\title{
Robust Stability Analysis of LCL Filter Based Synchronverter Under Different Grid Conditions
}

\author{
Roberto Rosso, Student Member, IEEE, Jair Cassoli, Giampaolo Buticchi, Senior Member, IEEE, Soenke \\ Engelken, Member, IEEE and Marco Liserre, Fellow, IEEE
}

\begin{abstract}
Synchronverters have gained interest due to their capability of emulating synchronous machines (SMs), offering self-synchronization to the grid. Despite the simplicity of the control structure, the adoption of an LCL-filter makes the overall model complex again, posing questions regarding the tuning of the synchronverter and its robustness. The multi-inputs multioutputs (MIMO) formulation of the problem requires multivariable analysis. In this paper, the effects of control parameter and grid conditions on the stability of the system are investigated by means of structured singular values (SSV or $\mu$-analysis). A stepby-step design procedure for the control is introduced based on a linearized small-signal model of the system. Then the design repercussions on the stability performance are evaluated through the performed robustness analysis. The developed linearized model is validated against time-domain simulations and laboratory experiments. These have been carried out using a power hardware-in-the-loop (PHIL) test bench, which allows to test the synchronverter under different grid conditions. As a conclusion the paper offers a simple guide to tune synchronverters but also a theoretical solid framework to assess the robustness of the adopted design.
\end{abstract}

Index Terms-Synchronverter robust stability analysis, $\mu$ analysis, synchronverter design, power hardware-in-the-loop tests.

\section{INTRODUCTION}

$\mathbf{T}$ HE amount of power electronics-based converters connected to the grid is growing noticeably causing concerns about the stability of the future power system. One of the main issues is related to the decrease of the total inertia of the system, but the risk of possible interactions between controllers of converters operating nearby cannot be underrated. Recent studies have shown that synchronization units of grid connected converters, usually phase-locked loops (PLLs), affect significantly the stability of the converters within their bandwidths [1]. Furthermore, interactions between synchronization units of power converters operating nearby have been observed and especially the fact that such effects are accentuated by weaker grid conditions [2]. During the last decade the concept of virtual synchronous machine (VSM) has been introduced [3]-[7]. Among the proposed control strategies, the synchronverter presented by Zhong et al. [5], [6] has been noted for its easy and intuitive structure and for

R. Rosso, J. Cassoli and S. Engelken are with Wobben Research and Development (WRD) GmbH, Borsigstr. 26, $26607 \mathrm{Au}$ rich, Germany (e-mail roberto.rosso@enercon.de, jair.cassoli@enercon.de, soenke.engelken@enercon.de).

G. Buticchi is with the University of Nottingham Ningbo China (UNNC), 199 Taikang East Road, 315100 Ningbo, China (e-mail buticchi@ieee.org.)

M. Liserre is with the University of Kiel, Kaiserstr. 2, 24143 Kiel, Germany (e-mailml@tf.uni-kiel.de). being the first proposed control algorithm in the literature for grid connected converters overcoming completely the need of a dedicated synchronization unit, both for initial synchronization to the main grid as well as during normal operation.

Synchronverter design has been recently addressed in several works [8]-[12]. Some of them rely on strong assumptions, such as grid short circuit ratio (SCR) higher then 10 in order to decouple active and reactive power loops [8]. Recent works presented tuning procedures based on reduced-order models of the system [10], [11]. Pole placement at prescribed locations in order to achieve desired dynamic has been proposed as optimal tuning procedure [11], [12] and eigenvalue analysis is the commonly adopted approach for investigation of stability of grid-connected converters and design purposes [13]. However, none of the works presented in the literature addresses robust stability analysis of the synchronverter. To this extent and according to the multi-inputs multi-outputs (MIMO) nature of the system, multivariable analysis is required [14], [15]. In fact, it is well known that eigenvalues are a poor measure of gain for MIMO systems, since they provide information about a specific system configuration and are not suitable for robust stability analysis and design of multivariable systems, where interactions between inputs and outputs of different channels take place [14].

It is common practice in the power electronics community to represent the grid as a Thévenin equivalent with a restiveinductive impedance, whose parameters are calculated according to the short circuit power at the point of common coupling (PCC) and the estimated $X / R$ ratio [16]. Unfortunately, this representation might be often inaccurate since grid conditions change substantially during the day, due to the presence of other converters operating nearby or due to the variation of the number and characteristics of connected loads. An efficient way for modelling such effects would be to include uncertainties on the nominal plant. In this scenario, the structured singular values (SSVs) analysis (commonly $\mu$-analysis) has been proven to be an efficient and reliable way for assessing robust stability of MIMO systems [14], [15], [17].

In this paper, the $\mu$-analysis is performed to assess the robust stability of an LCL grid connected synchronverter. The model developed in [10], based on linearized equations of the system, is adopted for the investigation. A design procedure of the synchronverter, using reduced-order models of active and reactive power loops is presented, which relys on nominal filter parameters and grid conditions. Subsequently, the $\mu$-analysis is performed according to a defined uncertainty function, in order to assess the effects of variations of control parameters 


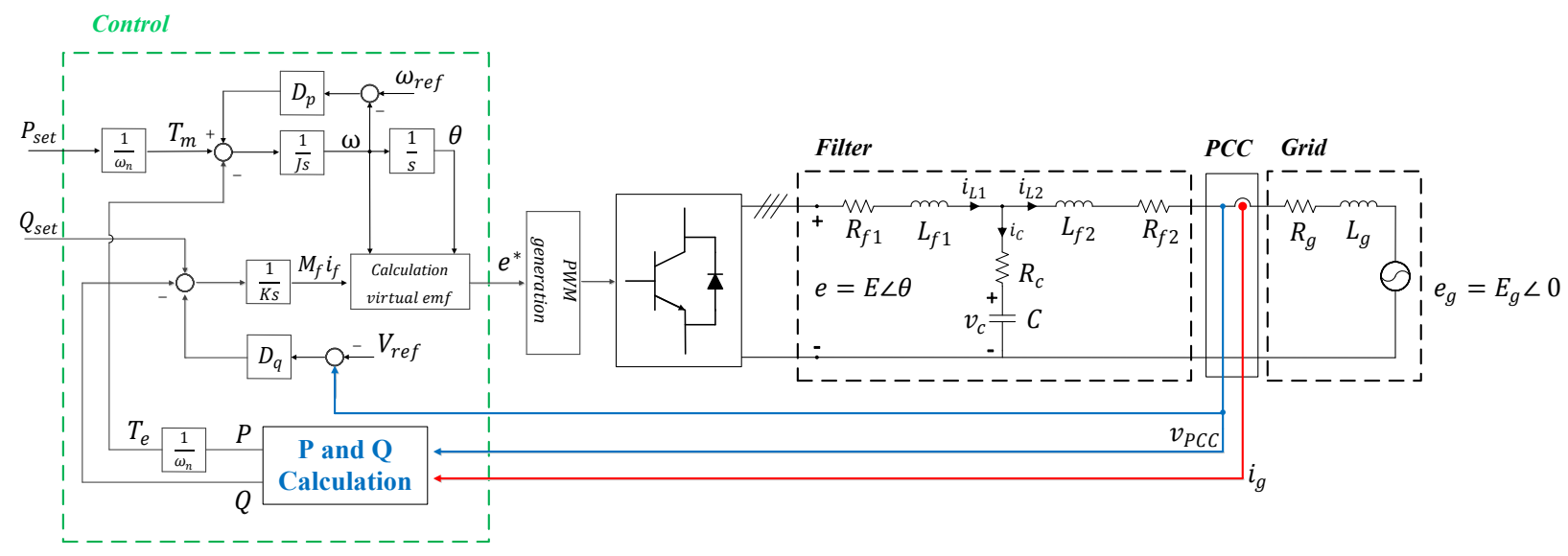

Fig. 1. Simplified scheme of the system under study.

and grid conditions on the stability of the system. Through the performed investigation effects that are not clearly visible by means of an eigenvalue analysis are highlighted, especially the fact that synchronverters turn to be very robust against grid uncertainties under weaker grid conditions, which is exactly the opposite trend shown by grid connected converters using dedicated synchronization units [2].

The rest of the paper is structured as follows: in section II, the small-signal model of a synchronverter connected to the grid through an LCL filter along with the adopted control design procedure are introduced. Section III presents the concept of robust stability analysis by means of SSVs and its application to the system under study. In section IV, experimental results using a power hardware-in-the-loop (PHIL) test bench are shown, while section $\mathrm{V}$ is dedicated to the conclusions.

\section{SMALl-Signal Model AND DESIGN}

In the following, the small-signal model of a synchronverter connected to the grid through an output LCL filter presented in [10] is briefly introduced.

The model has been developed by splitting the overall system into two separated subsystems, namely the control and the plant composed of the filter and the grid. The simplified scheme of the system under study is depicted in Fig. 1, while in Fig. 2, inputs and outputs of the two linearized subsystems are shown. The design procedure presented in [10] is described in this section and is adopted in this work for tuning the parameters of the control.

\section{A. Small-signal model}

The synchronverter control structure shown in Fig. 1 contains separated loops for active power $P$ and a reactive power

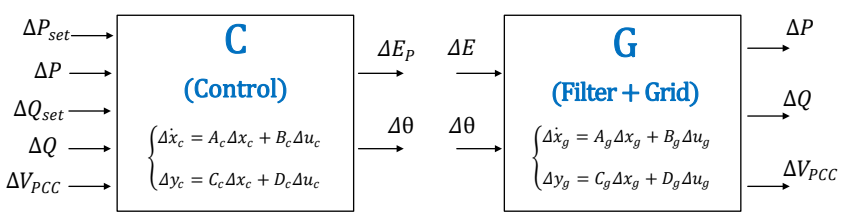

Fig. 2. Inputs and outputs of the two linearized subsystems.
$Q$. The former emulates the frequency droop mechanism typical of a SM described by the well-known swing equation:

$$
J \dot{\omega}=T_{m}-T_{e}-D_{p} \omega,
$$

with $J$ representing the mechanical (virtual) inertia, $\omega$ the virtual rotor speed, $D_{p}$ the feedback gain accounting for the (virtual) mechanical friction of the machine, $T_{e}$ is the electrical torque and $T_{m}$ is the mechanical one. $D_{p}$ does not only represent the virtual friction of the machine, but also the active power-frequency droop coefficient of the controller. $T_{m}$ can be directly calculated from the active power setpoint $P_{\text {set }}$ simply by dividing by the nominal frequency $\omega_{n}$. The virtual rotor angle $\theta$ is obtained integrating $\omega$ and is needed for the calculation of the virtual back-emf $e^{*}$. The reactive powervoltage droop control reacts to a voltage deviation $\Delta_{V}$ from its nominal/reference value with a change of the reactive power setpoint $\Delta_{Q}$, according to the droop coefficient $D_{q}$ :

$$
\Delta_{Q}=-D_{q} \Delta_{V}
$$

The instantaneous reactive power measured at the output of the converter is then compared to its setpoint and added to the signal coming from the voltage droop. The resulting quantity is processed through an integrator with gain $1 / K$ producing the virtual mutual flux $M_{f} i_{f}$, which multiplied by the virtual rotor speed $\omega$ produces the amplitude of the virtual back-emf $E_{p}$. Linearization of this product yields:

$$
\Delta E_{p}=M_{f} i_{f 0} \Delta \omega+\Delta M_{f} i_{f} \omega_{0},
$$

where quantities with subscript " 0 " denote values at the operating point. The chosen state variables of the control system are the virtual rotor rotational speed $\omega$, the rotor angle $\theta$ and the mutual flux $M_{f} i_{f}$.

The state-space representation of the plant composed of the filter and the grid is obtained by choosing $i_{L 1}, i_{L 2}$ and $v_{c}$ of Fig. 1 as state variables, namely filter current at converter side, filter current at grid side and capacitor voltage, respectively, and writing the equations in $d q$ coordinates. Linearization is required for the calculation of active and reactive power as well as for the voltage amplitude at the PCC: 


$$
\left\{\begin{array}{c}
\Delta P=\frac{3}{2}\left(I_{L 2 d 0} \Delta v_{P C C d}+V_{P C C d 0} \Delta i_{L 2 d}+\right. \\
\left.+V_{P C C q 0} \Delta i_{L 2 q}+I_{L 2 q 0} \Delta v_{P C C q}\right) \\
\Delta Q=\frac{3}{2}\left(V_{P C C q 0} \Delta i_{L 2 d}+I_{L 2 d 0} \Delta v_{P C C q}+\right. \\
\left.-V_{P C C d 0} \Delta i_{L 2 q}+I_{L 2 q 0} \Delta v_{P C C d}\right) \\
\Delta V_{P C C}=\frac{V_{P C C d 0} \Delta v_{P C C d}+V_{P C C q 0} \Delta v_{P C C q}}{\sqrt{V_{P C C d 0}^{2}+V_{P C C q 0}^{2}}}
\end{array},\right.
$$

where $\Delta v_{P C C d}$ and $\Delta v_{P C C q}$ can be calculated as:

$$
\left\{\begin{array}{c}
\Delta v_{P C C d}=\Delta v_{c d}+R_{c}\left(\Delta i_{L 1 d}-\Delta i_{L 2 d}\right)+ \\
-R_{f 2} \Delta i_{L 2 d}-L_{f 2} \frac{d i_{L 2 d}}{d t}+\omega_{0} L_{f 2} \Delta i_{L 2 q} \\
\Delta v_{P C C q}=\Delta v_{c q}+R_{c}\left(\Delta i_{L 1 q}-\Delta i_{L 2 q}\right)+ \\
-R_{f 2} \Delta i_{L 2 q}-L_{f 2} \frac{d i_{L 2 q}}{d t}-\omega_{0} L_{f 2} \Delta i_{L 2 d}
\end{array}\right.
$$

where $R_{f 2}$ and $L_{f 2}$ represent the resistance and inductance of the grid-side elements of the filter, respectively, whereas $R_{c}$ is the capacitor damping resistance.

\section{B. Control Design}

The design procedure adopted in this work aims to the optimization of the step response characteristics of the system in terms of rise time, overshoot and settling time. Its intent is to provide a simple and intuitive approach for the design of the synchronverter when the characteristics of the filter and the grid are known, avoiding the designer to rely on a trial and error procedure. It considers active and reactive power loops separately and approximates the corresponding closedloop transfer functions to simplified second-order equations. Control parameters are calculated setting a desired damping factor. In fact, it is known from control theory that the optimal dynamic response of a second-order system is obtained setting the damping ratio to $1 / \sqrt{2}$ [19]. Therefore, control parameters are simply calculated expressing the equivalent damping ratios of the reduced-order closed loop transfer functions in terms of plant and control parameters and chosen so as to obtain the desired damping ratio. Recently, another work on synchronverter design based on a reduced-order model has been presented in [11]. It is important to point out that the introduced simplifications may result in design errors, with repercussions on the performance of the system. In fact, as it will be shown in the following subsection, it is always reccomended to check the dynamic performances of the system using a full-order model to be sure that they comply with the required specifications. Regarding stability assessment, since the two loops are considered separately and due to the MIMO nature of the system under study, even using the full-order transfer functions of the plant in the active and reactive power loops design might lead to erroneous results [14], [15], due to the fact that the cross-coupling effects between the two loops are neglected. For this reason, multivariable systems theory should be applied in order to properly design the control. In the next section, the $\mu$-analysis is performed in order to assess the stability margin of the MIMO system under study against a defined set of plant uncertainties, once the control has been tuned according to the adopted design procedure. Subsequently, the effects of control parameters variations on

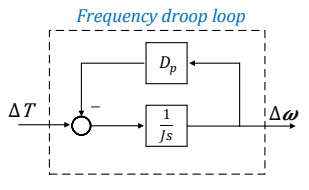

(a)

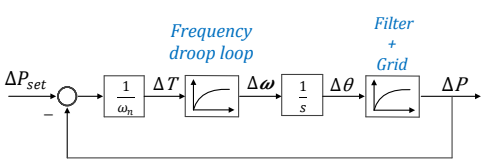

(b)
Fig. 3. (a) Simplified frequency droop loop, (b) simplified active power loop.

the stability of the system are estimated, such that the designer can find the most suitable compromise between dynamic performances and stability margin. In [10], three different cases with different grid and filter characteristics have been examined, in order to test the effectiveness of the proposed design procedure under different system conditions. In this paper, one of the cases examined in [10], whose parameters are reported in Table $\mathrm{I}$, is taken as reference and used in the following to describe the adopted design procedure. In this work, the filter design is not explicitly addressed and filter parameters are obtained according to the filter design procedure presented in [18].

The parameters to be tuned are the $P-f$ droop coefficients $D_{p}$ and the virtual inertia $J$ for the active power loop along with the $Q-V$ droop coefficient $D_{q}$ and the factor $K$ of the reactive power loop. Often droop coefficients are already fixed due to specifications on the steady-state response and therefore only $J$ and $K$ can be adjusted to improve the dynamic behaviour. However, the adopted design procedure is valid even in case that the four parameters are freely adjustable.

In Fig. 3(a), the simplified scheme of the synchronverter frequency droop loop is shown, described by the following first-order transfer function:

$$
\frac{\Delta \omega}{\Delta T}(s)=\frac{\frac{1}{D_{p}}}{1+s \frac{J}{D_{p}}}=\frac{K_{f}}{1+s \tau_{f}} .
$$

In Fig. 3(b), the simplified scheme of the active power closed-loop is reported. The plant composed of the filter and the grid (indicated as $G$ in Fig. 2) is described dynamically by the transfer function $\frac{\Delta_{P}}{\Delta_{\theta}}$. Each of the input-output transfer functions of $G$ can be approximated by an equivalent firstorder transfer function by looking at the characteristics of the poles of the system, shown in Table II for simplicity. Two time constants identify the dynamic of the system, indicated as $\tau_{p 1}$ and $\tau_{p 2}$ in Table II. The transfer function $\frac{\Delta_{P}}{\Delta_{\theta}}$ of $G$ can be approximated to the following first-order transfer function:

$$
P T 1_{P}(s)=\frac{G_{p}}{1+s \tau_{r e f p}},
$$

where $G_{P}$ is the steady-state value of $\frac{\Delta_{P}}{\Delta_{\theta}}$ and $\tau_{r e f p}$ the time constant of its dominant pole, namely $\tau_{p 1}$ for this case, as can be determined by observing its step response. Choosing $J$ sufficiently small (e.g. $\tau_{f} \approx \tau_{r e f p} / 10$ ), the frequency droop loop can be neglected and the active power loop can be approximated by the second-order transfer function reported below: 
Table I

PARAMETERS OF THE EXAMINED CASE

\begin{tabular}{ccc}
\hline \hline Description & Symbol & Value \\
\hline Inverter rated power & $S_{n}(\mathrm{kVA})$ & 300 \\
Short circuit ratio & $S C R$ & 20 \\
Reactive-resistive ratio & $X / R$ & 10 \\
Line-to-line voltage & $V_{L L}(\mathrm{Vrms})$ & 400 \\
Rated grid frequency & $f_{g}(\mathrm{~Hz})$ & 50 \\
Grid inductance & $L_{g}(\mathrm{pu})$ & 0.05 \\
Inverter-side filter inductor & $L_{f 1}(\mathrm{pu})$ & 0.08 \\
Grid-side filter inductor & $L_{f 2}(\mathrm{pu})$ & 0.02 \\
Grid resistance & $R_{g}(\mathrm{pu})$ & 0.005 \\
Inverter-side filter resistor & $R_{f 1}(\mathrm{pu})$ & 0.02 \\
Grid-side filter resistor & $R_{f 2}(\mathrm{pu})$ & 0.02 \\
Capacitor damping resistor & $R_{c}(\mathrm{pu})$ & 0.18 \\
Filter capacitor & $C(\mathrm{pu})$ & 0.05 \\
\hline \hline
\end{tabular}

Table II

CHARACTERISTICS OF THE POLES OF $G$

\begin{tabular}{ccccc}
\hline \hline Pole & Damping & $\begin{array}{c}\text { Frequency } \\
(\mathrm{rad} / \mathrm{sec})\end{array}$ & $\begin{array}{c}\text { Time constant } \\
(\mathrm{sec})\end{array}$ \\
\hline$\left(p_{1}-p_{2}\right)$ & $-94.8 \pm j 314$ & 0.289 & 328 & $1.05 \mathrm{e}-2\left(\tau_{p 1}\right)$ \\
$\left(p_{3}-p_{4}\right)$ & $-842 \pm j 6930$ & 0.120 & 6980 & $1.19 \mathrm{e}-3\left(\tau_{p 2}\right)$ \\
$\left(p_{5}-p_{6}\right)$ & $-842 \pm j 7560$ & 0.111 & 7610 & $1.19 \mathrm{e}-3\left(\tau_{p 2}\right)$ \\
\hline \hline
\end{tabular}

$$
P_{a p p}(s)=\frac{1}{T_{p}^{2} s^{2}+2 \zeta_{p} T_{p} s+1},
$$

where $T_{p}$ and $\zeta_{p}$ represent the inverse natural frequency and the damping ratio, respectively:

$$
T_{p}=\sqrt{\frac{\tau_{r e f p} \omega_{n} D_{p}}{G_{p}}} \quad ; \quad \zeta_{p}=\frac{1}{2} \sqrt{\frac{D_{p} \omega_{n}}{\tau_{r e f p} G_{p}}} .
$$

Assuming $D_{P}$ bounded in order to comply with steadystate performance requirements, the damping of the simplified second-order active power loop transfer function cannot be influenced otherwise [7]. In case that $D_{P}$ is freely adjustable, it can be tuned so as to achieve $\zeta_{P}=1 / \sqrt{2}$.

A Similar approach can be adopted for the tuning of the reactive power loop. Due to the $Q-V$ droop, two different loops are identified, namely a reactive power and a voltage control loop, shown in Fig. 4(a) and (b) respectively.

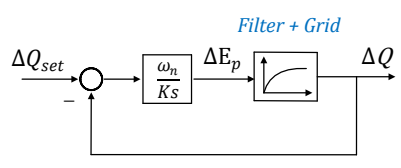

(a)

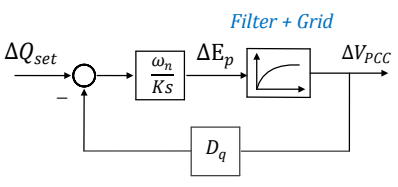

(b)
Fig. 4. (a) Simplified reactive power loop scheme, (b) simplified $V_{P C C}$ loop scheme.

Also in this case, the behaviours of $\frac{\Delta Q}{\Delta E_{p}}$ and $\frac{\Delta V_{P C C}}{\Delta E_{p}}$ of $G$ are approximated by reduced first-order transfer functions indicated as $P T 1_{Q}$ and $P T 1_{V}$, respectively:

$$
P T 1_{Q}(s)=\frac{G_{q}}{1+s \tau_{r e f q}} \quad ; \quad P T 1_{V}(s)=\frac{G_{v}}{1+s \tau_{r e f v}},
$$

where $G_{q}$ and $G_{v}$ are the steady-state values of $\frac{\Delta_{Q}}{\Delta E_{p}}$ and
$\frac{\Delta V_{P C C}}{\Delta E_{p}}$, respectively and $\tau_{\text {ref } Q}$ and $\tau_{\text {refV }}$ are the time constants of the respective dominant poles of each transfer function. The resulting damping factors of the two approximated transfer functions are reported below:

$$
\zeta_{q}=\frac{1}{2} \sqrt{\frac{K}{\tau_{r e f q} \omega_{n} G_{q}}} \quad ; \quad \zeta_{v}=\frac{1}{2} \sqrt{\frac{K}{\tau_{r e f v} \omega_{n} D_{q} G_{v}}} .
$$

If $D_{q}$ can be arbitrarily modified, $K$ and $D_{q}$ can be chosen such that $\zeta_{q}=\zeta_{v}=1 / \sqrt{2}$, otherwise the highest value of $K$ resulting from the two calculations is chosen.

Assuming that $D_{p}$ and $D_{q}$ are fixed to $5 \%$ [20], the calculated control parameters $J_{o p t}$ and $K_{o p t}$ for the case under study are reported in Table III.

Table III

\begin{tabular}{cc}
\multicolumn{2}{c}{ CONTROL PARAMETERS } \\
\hline \hline Parameter & Value \\
\hline$D_{p}$ & 60.8 \\
$D_{q}$ & 18371 \\
$J_{o p t}$ & $6.38 \mathrm{e}-2$ \\
$K_{\text {opt }}$ & 37459 \\
\hline \hline
\end{tabular}

\section{Simulation Results}

Simulation results obtained using the full-order model of the system and setting the values of the control parameters accordingly to the adopted design procedure are shown in Fig. 5. Active and reactive power loops have been tested separately and sweeps of $J$ and $K$ are performed. Firstly, the response of the system to a step of $P_{\text {set }}$ of 0.3 pu was observed varying the value of $J$ within the range $\left[\frac{J_{o p t}}{50} ; 50 J_{o p t}\right.$, whereas $K$ was set to the calculated optimal value $K_{\text {opt }}$. Subsequently, the response of the system to a step $Q_{\text {set }}$ of the same amplitude was simulated varying $K$ within the range $\left[\frac{K_{o p t}}{5} ; 5 K_{o p t}\right]$, whereas $J=J_{o p t}$.

The directions of the arrows indicate the increment of the corresponding parameters, the red curve is the response obtained by setting the parameters calculated using the adopted design procedure, whereas green curves are for values below the calculated optimal one and blue curves for values above it. In Fig. 5 (b), the steady-state value of the reactive power does not reach the given setpoint of 0.3 pu due to the voltage droop controller, which adjusts the reactive power according to the measured voltage at the PCC.

\section{ROBUST STABILITY ANALYSIS}

Most of the works in the literature regarding synchronverter design are based either on analysis of linearized system transfer functions [8], [10], or eigenvalue analysis [11], [12]. Although eigenvalue analysis is an efficient way for assessing system stability, it is well known that for MIMO systems eigenvalues are a poor measure of gain [14]. Indeed, eigenvalues provide only information about a specific configuration of the system when inputs and outputs are in the same direction, namely the direction of the eigenvectors. They do not take into 

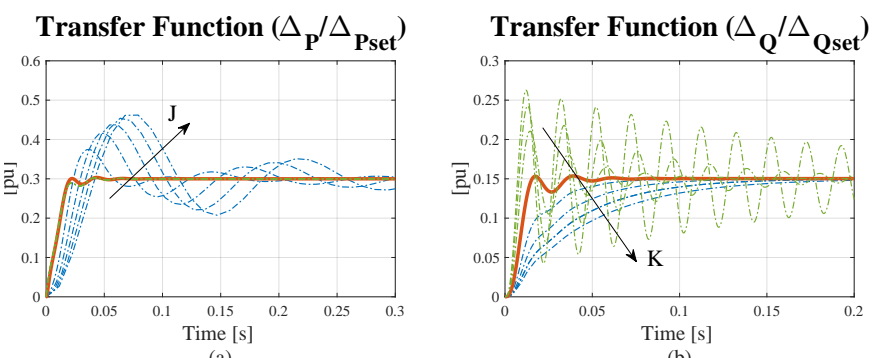

Fig. 5. (a) Effects of $J$ on the dynamic behaviour of $\frac{\Delta_{P}}{\Delta P_{\text {set }}}$; (b) effects of $K$ on the dynamic behaviour of $\frac{\Delta_{Q}}{\Delta Q_{s e t}}$. (red): Response with the parameters from design procedure, (green): response for values lower then the optimal one (blue): response for values higher then the optimal one.

account the possible interactions between different channels that typically occur in a MIMO system. For accurate robustness assessment of MIMO systems, multivariable analysis is required and different methods have been developed, such as the structured singular values (SSV) or $\mu$-analysis [14], [15], [17]. Singular values provide better information about the gains of the plants and the robustness of the control is verified against a defined set of system uncertainties. This method allows to span a set of possible system configuration instead of verifying stability only for a specific condition.

Parameter inaccuracies or non-linearities are among the most common sources of uncertainties. Other common sources of uncertainties are represented by neglected dynamics due to delays of sensors and/or actuators. These kind of uncertainty sources have a frequency dependent behaviour and can be included in the set of uncertain plants by using appropriate representations. The control loops of each grid connected converter modify the frequency behaviour of the equivalent grid seen by other converters operating nearby [13]. These effects can be also included in the stability analysis as uncertainties on the plant. The common way of modelling uncertainties is to add perturbations to the nominal plant $G$ in an additive or multiplicative way [14], [15]. The multiplicative representation consists on defining the set of perturbed plants as:

$$
G_{p}=G(1+w \Delta) ; \text { with }\|\Delta\|_{\infty} \leq 1 ;
$$

where $\Delta$ is a block diagonal normalized matrix including all the possible perturbations, $\|\Delta\|_{\infty}$ represents its $\mathcal{H}_{\infty}$ norm and $w$ is a multiplicative weight. The multiplicative representation is often preferred over the additive one, as the numerical values of its weights are more informative [14]. For example, at frequencies where $|w(j \omega)|>1$, the uncertainty exceeds $100 \%$. Multiplicative uncertainties are characterized by a small amplitude for low frequency, increasing to unity and above at higher frequencies. This is a consequence of dynamic properties that inevitably occur in physical systems. Uncertainties might be located at the input or at the output of the plant. Input multiplicative uncertainty is suitable for modeling uncertain high frequency dynamics and uncertain right half plane zeros, while output inverse multiplicative uncertainty for low frequency parameter errors and uncertain right half plane poles [15]. In this work, the input multiplicative uncertainty representation is used in order to investigate the robustness of the control against high-order frequency effects, such as resonant grid impedance behaviour or the effects of controllers of other power electronics converters operating nearby. A multiplicative uncertainty weight is defined and, according to it, the robustness of the control is investigated varying controller parameters and grid conditions. The results are then compared to eigenvalue analysis, showing that the analysis performed with structured singular values enables highlighting effects that cannot be explicitly observed looking at the eigenvalues of the system.

\section{A. Problem formulation for $\mu$-analysis}

In order to perform the $\mu$-analysis, the system should be represented in the lower fractional transformation (LFT) form [17]. This process is often referred to as "pulling out the $\Delta$ 's" [15]. In Fig. 6 the steps required for bringing the system in a form suitable for $\mu$-analysis are shown. First, the uncertainties have to be "pulled out" from the plant and the system has to be put in the form shown in Fig. 6 (a). Subsequently, the $N \Delta$ structure shown in Fig. 6 (b) is obtained by means of a LFT between the generalized plant $P$ and the controller $C$, defined as [14]:

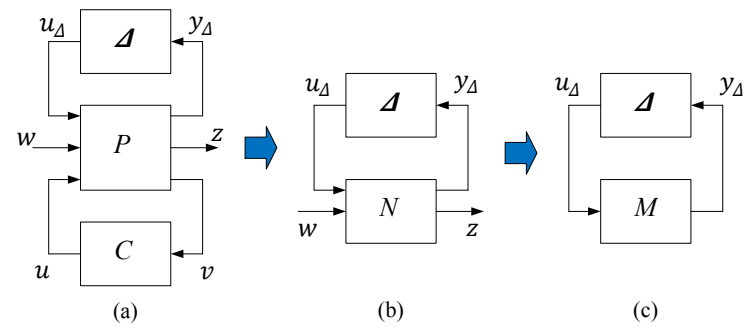

Fig. 6. Problem formulation for $\mu$-analysis: (a) general control formulation, (b) $\mathrm{N} \Delta$-structure, (c) $\mathrm{M} \Delta$-structure.

$$
N=F_{l}(P, C) \triangleq P_{11}+P_{12} C\left(I-P_{22} C\right)^{-1} P_{21} .
$$

Finally, the $M \Delta$-structure is simply derived considering that $M=N_{11}$. The structured singular value $\mu$ is defined as the smallest structured $\Delta$ (measured in terms of the largest singular value $\bar{\sigma}(\Delta)$ ), which makes the matrix $I-M \Delta$ singular; then [14]:

$$
\mu(M) \triangleq \min _{\Delta}\{\bar{\sigma} \mid \operatorname{det}(I-M \Delta)=0 \text { for structured } \Delta\} .
$$

The inverse of $\mu(M)$ can be interpreted as a stability margin with respect to the structured uncertainty set affecting $M$. This means that indicating the peak of $\mu(M)=\beta$ across the frequency range $\omega$, stability is guaranteed for all perturbations with appropriate structure and with respect to the chosen uncertainty, such that:

$$
\max _{\omega} \bar{\sigma}(\Delta) \leq \frac{1}{\beta} .
$$

It is therefore clear that the resulting stability margin is very related to the uncertainties considered for the analysis. 


\section{B. Application to the system under study}

A practical application of $\mu$-analysis to the studied system is presented in the following. All the required calculations can be carried out by means of the "Control System Toolbox" of MATLAB [21]. The generalized plant $P$ shown in Fig. 6 (a) can be obtained using the command sysic. The general control formulation for the particular case under study is shown in Fig. 7, where $y_{\Delta}=\left[\begin{array}{ll}z_{1} & z_{2}\end{array}\right]^{T}, u_{\Delta}=\left[\begin{array}{ll}w_{1} & w_{2}\end{array}\right]^{T}$ and the two multiplicative input uncertainties $W_{\delta 1}$ and $W_{\delta 2}$ have been located at the input channels $u_{1}$ and $u_{2}$. Outputs of $P$ are then the control inputs shown in Fig. 2 and represented by the vector $v=\left[\begin{array}{lllll}v_{1} & v_{2} & v_{3} & v_{4} & v_{5}\end{array}\right]$. Using the starp command from the same toolbox of MATLAB, the star product between $P$ and $C$ can be calculated, obtaining (13).

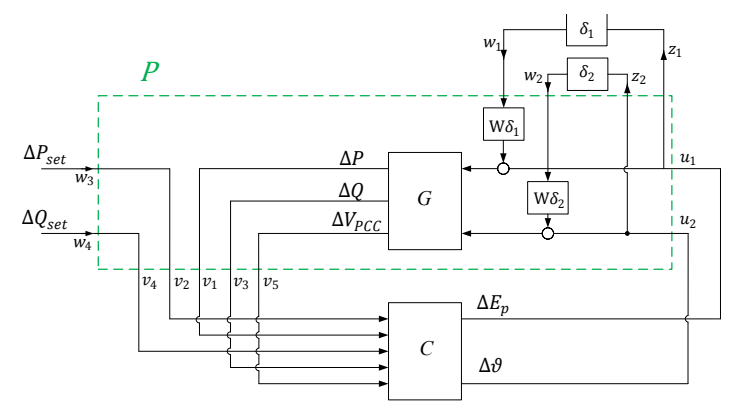

Fig. 7. Construction of the generalized plant of the system under study.

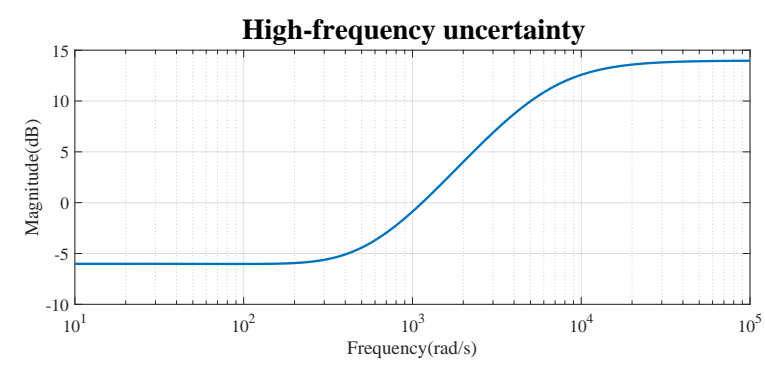

Fig. 8. Multiplicative input uncertainty used for the $\mu$-analysis.

In Fig. 8, the multiplicative input uncertainty used for the analysis is reported. It shows an amplitude of $50 \%$ at low frequency, increasing till $500 \%$ at very high frequencies. The chosen weight accounts for low frequency uncertainties due to parametric uncertainty in the model as well as high frequency neglected dynamic effects or resonant effects of the grid due to the presence of other converters operating nearby. Considering the system with the parameters listed in Table I and Table III, in the following the effects of control parameters $J$ and $K$ as well as grid $S C R$ variations on the eigenvalues of the systems are shown and compared to the results obtained through the $\mu$-analysis.

\section{Variation of $\mathbf{J}$}

In Fig. 9 (a) and (b), the migration of the nine eigenvalues of the full-order system due to a sweep of $J$ in the range $\left[\frac{J_{o p t}}{20} ; 20 J_{\text {opt }}\right]$ is shown. The direction of the arrows indicate
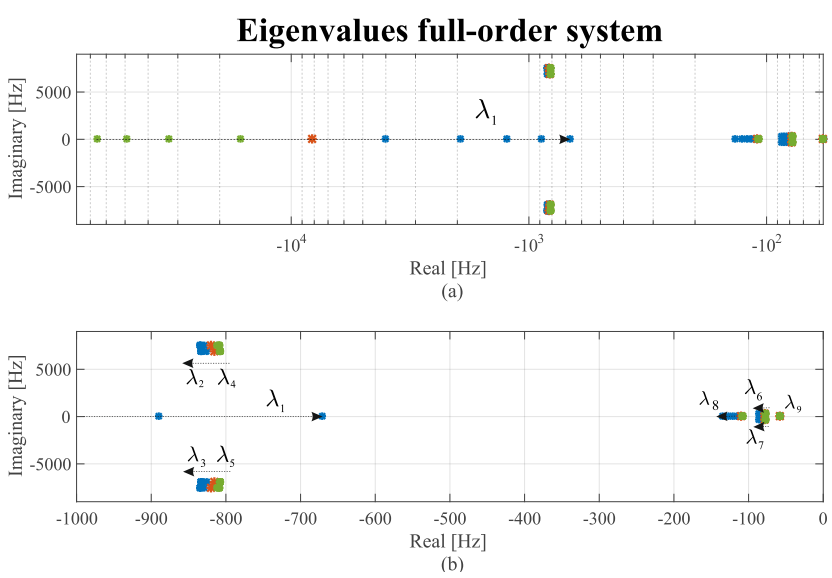

Fig. 9. Effects of variation of control parameter $J$ on the eigenvalues of the system, (a) migration of $\lambda_{1}$, (b) migration of $\lambda_{2}-\lambda_{9}$.

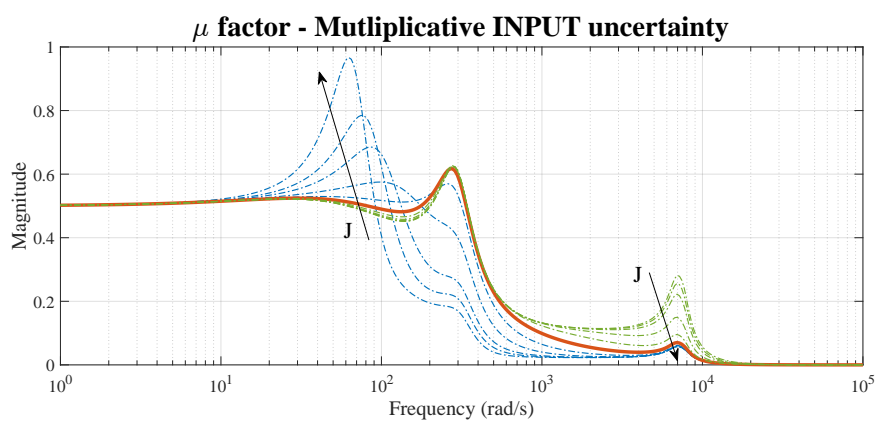

Fig. 10. Behaviour of $\mu$ factor due to variations of $J$.

the increase of the parameter $J$. Red points indicate the locations of the eigenvalues for reference conditions represented by $J=J_{o p t}, K=K_{\text {opt }}$ and $S C R=20$, whereas green points are for values of $J<J_{\text {opt }}$ and blue ones for values of $J>J_{\text {opt }}$. It is clear that the parameter $J$ is mainly affecting the eigenvalue $\lambda_{1}$, causing a migration of the eigenvalue toward the imaginary axis, whereas the other eight eigenvalues move leftwards.

In Fig. 10, the results of the $\mu$-analysis are shown, where colours have the same meaning as in Fig. 9. Under reference conditions, the control is quite robust against the defined plant uncertainties. The maximum value of $\mu=0.6169$ and is reached at a frequency of $277 \mathrm{rad} / \mathrm{sec}$. According to the results shown in Fig. 10, an increase of $J$ tends to augment the robustness of the system at higher frequency, as can be also deduced from the eigenvalue analysis, since the dominant eigenvalues move leftwards. However, an excessive increase of this parameter increases the peak of the $\mu$ factor at lower frequencies, reaching almost the unity for $J=20 J_{\text {opt }}$. This represents an important aspect that needs to be considered during the design procedure. In fact, it is common thought that VSMs should reproduce the inertia of a real synchronous machine of the same size. This requires the introduction of additional energy storage in the DC-Link of the converter, increasing sizes and costs. The results shown through this analysis indicate that generally high values of virtual inertia $J$ do not always correspond to an increment of the stability margin of the converter. 

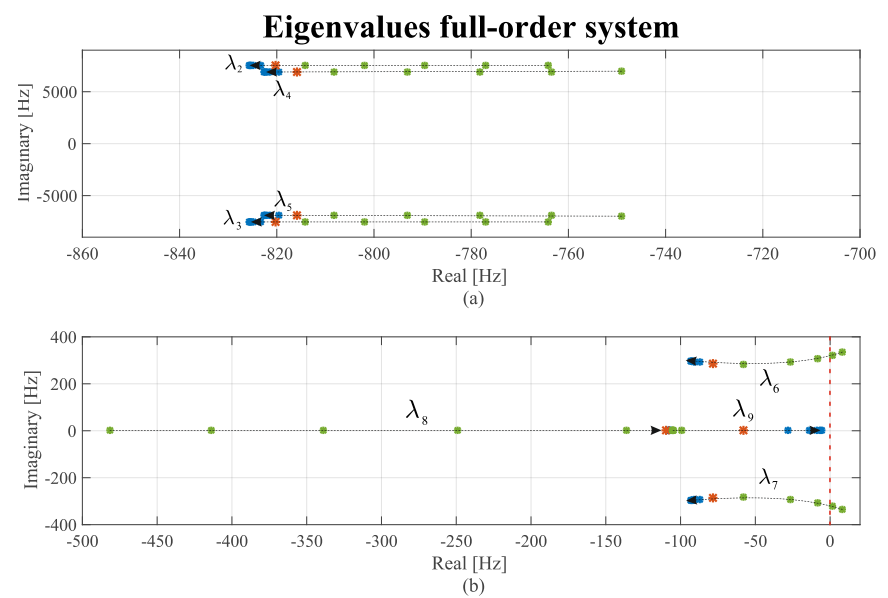

Fig. 11. Effects of variation of control parameter $J$ on the eigenvalues of the system, (a) migration of $\lambda_{2}-\lambda_{5}$, (b) migration of $\lambda_{6}-\lambda_{9}$.

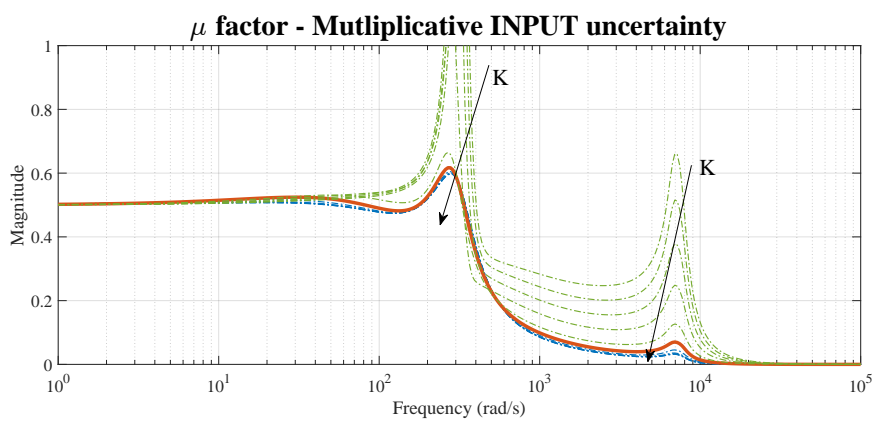

Fig. 12. Behaviour of $\mu$ factor due to variations of $K$.

\section{Variation of $K$}

Fig. 11 (a) and (b) show the migration of the eigenvalues due to variation of the parameter $K$. The parameter is varied within the range $\left[\frac{K_{o p t}}{5} ; 5 K_{o p t}\right] . \lambda_{1}$ is far away from the origin and is not relevantly affected. For values of $K$ lower then $K_{\text {lim }} \approx 9000, \lambda_{6}$ and $\lambda_{7}$ are located on the right half plane causing instability.

The results of the $\mu$-analysis shown in Fig. 12 indicate an increase of the robustness of the system for higher values of $K$ at all frequencies. However, for values of $K>K_{o p t}$ the stability margin is not relevantly enhanced. It is worth pointing out that, according to the chosen uncertainty weights, the limit of $\mu=1$ is reached for $K \approx 19000$, which is more conservative if compared to the limit obtained through the eigenvalue analysis. This is simply explained by the fact that $\mu$ provides information about robustness, covering a wide set of possible plants, while eigenvalues show results only for one particular system.

\section{E. Variation of grid SCR}

Fig. 13 shows the migration of the eigenvalues due to a decrease of the grid $S C R$ from 20 to 2 , when the $X / R$ ratio is set to 10 . The direction of the arrows indicates the increase of the corresponding value, showing clearly that all the eigenvalues move rightwards when the impedance of the
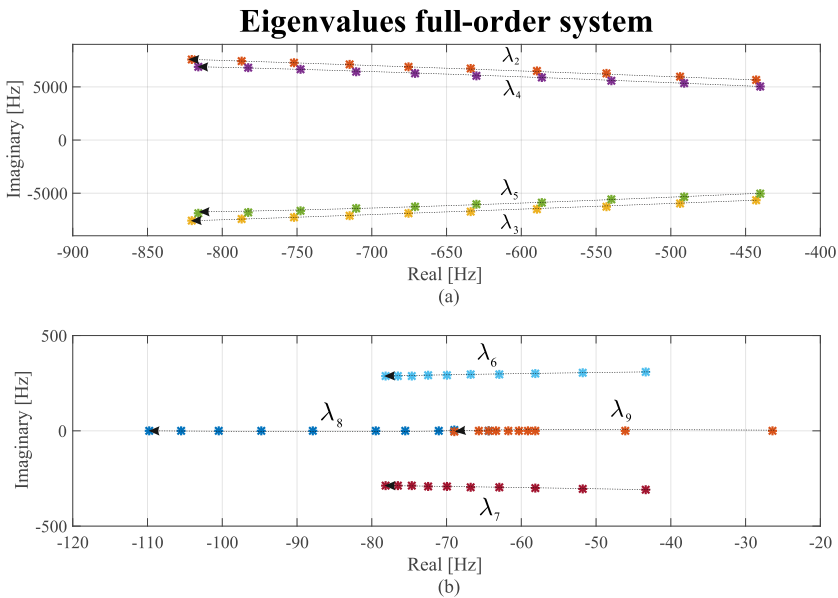

Fig. 13. Effects of variation of grid SCR on the eigenvalues of the system, (a) migration of $\lambda_{2}-\lambda_{5}$, (b) migration of $\lambda_{6}-\lambda_{9}$.

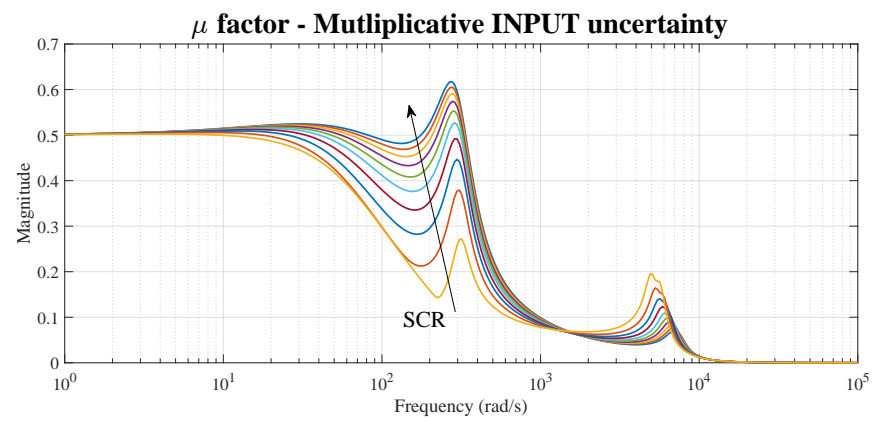

Fig. 14. Behaviour of $\mu$ factor due to variations of the grid SCR.

grid is increased. This would lead to the consideration that the synchronverter is less stable when connected to a weaker grid. Similar conclusions have been drawn in [22], where the stability of a synchronverter-dominated microgrid has been investigated.

However, looking at the results of the $\mu$-analysis reported in Fig. 14, the decrease of the grid $S C R$ has instead the effect of reducing the $\mu$ factor for all frequencies below $\approx 1000 \mathrm{rad} / \mathrm{sec}$, where the highest peak of $\mu$ is located. This indicates that the control results to be more robust against the defined set of uncertainties shown in Fig. 8 when connected to a grid with higher impedance. This can be explained by the fact that the synchronverter behaves as a SM and basically as a voltage source behind an impedance. Indeed, stator inductances of SMs are usually much higher than typical converter filter inductances and their high impedance enhances the stability of the machine against high frequency perturbations [23].

It is worth to notice that the behaviour of the synchronverter highlighted through the performed analysis contrasts the trend of PLL-based converters, which instead result to be much more sensitive to perturbations in the plant (represented for example by the presence of other converters operating nearby) when connected to a weaker grid [2].

\section{F. Considerations for synchronverter design}

According to the results of the robust stability analysis performed in this section, the following aspects should be 
taken into account during the design procedure:

- In this work, control parameters have been tuned without considering any requirements on virtual inertia. In fact, in the design procedure presented in the previous section, $J$ has been set to a proper low value so as to neglect the dynamic of the frequency droop loop in the active power control loop. For the specific case under study, a stability margin improvement is achieved for values of $J$ up to $\approx 10 J_{o p t}$. However, a further increase of $J$ causes a reduction of the stability margin of the converter at low frequencies.

- $K$ affects significantly the stability of the converter. To comply with the design procedure presented in the previous section, the highest value of $K$ resulting from (11) has been selected as $K_{o p t}$. According to the results of the performed stability analysis and observing the dynamic behaviour shown in Fig. 5 (b), a further increase of $K$ over a certain limit simply worsen the dynamic performances of the converter, without significantly improving its stability.

- The performed analysis has pointed out that the stability of the synchronverter against the defined set of high frequency uncertainties is augmented by the presence of high impedance between the converter and the grid. As already mentioned, stator inductances of SMs are much higher than usual converter filter inductances, typically designed so as to optimize the trade-off between power quality and size of the filter components [18]. A simple and efficient way for reproducing the characteristics of a SM by means of a VSC without necessarily increasing the size of the hardware components, is the emulation of virtual impedance through the control [23]. The use of virtual impedances is well-known in the literature and several techniques have been proposed for various purposes, e. g. harmonic suppressions [24], inverter currents limitation [25] or output admittance shaping [26], to name but a few. An overview on the techniques proposed in the literature is not the focus of this work, but rather highlighting that this expedient might be adopted in order to enhance the stability of the synchronverter [23].

Finally, one might think about including the stability analysis shown in this paper as part of a comprehensive design procedure, which aims to find the best compromise between dynamic performances and stability margin. An example of a possible iterative process is reported in Fig. 15. The flowchart shown in Fig. 15 (a) summarizes the steps of the design method adopted in this paper and described in section II.B, whereas in Fig. 15 (b), the extended flowchart including stability margin considerations is depicted.

\section{EXPERIMENTAL RESULTS}

Laboratory experiments have been performed in order to validate the linearized model used for the analysis. In Fig. 16 (a), a simplified scheme of the experimental setup used for the tests is depicted, while in Fig. 16 (b), a picture of the laboratory environment is shown. Each phase of a $4 \mathrm{kVA}$ converter

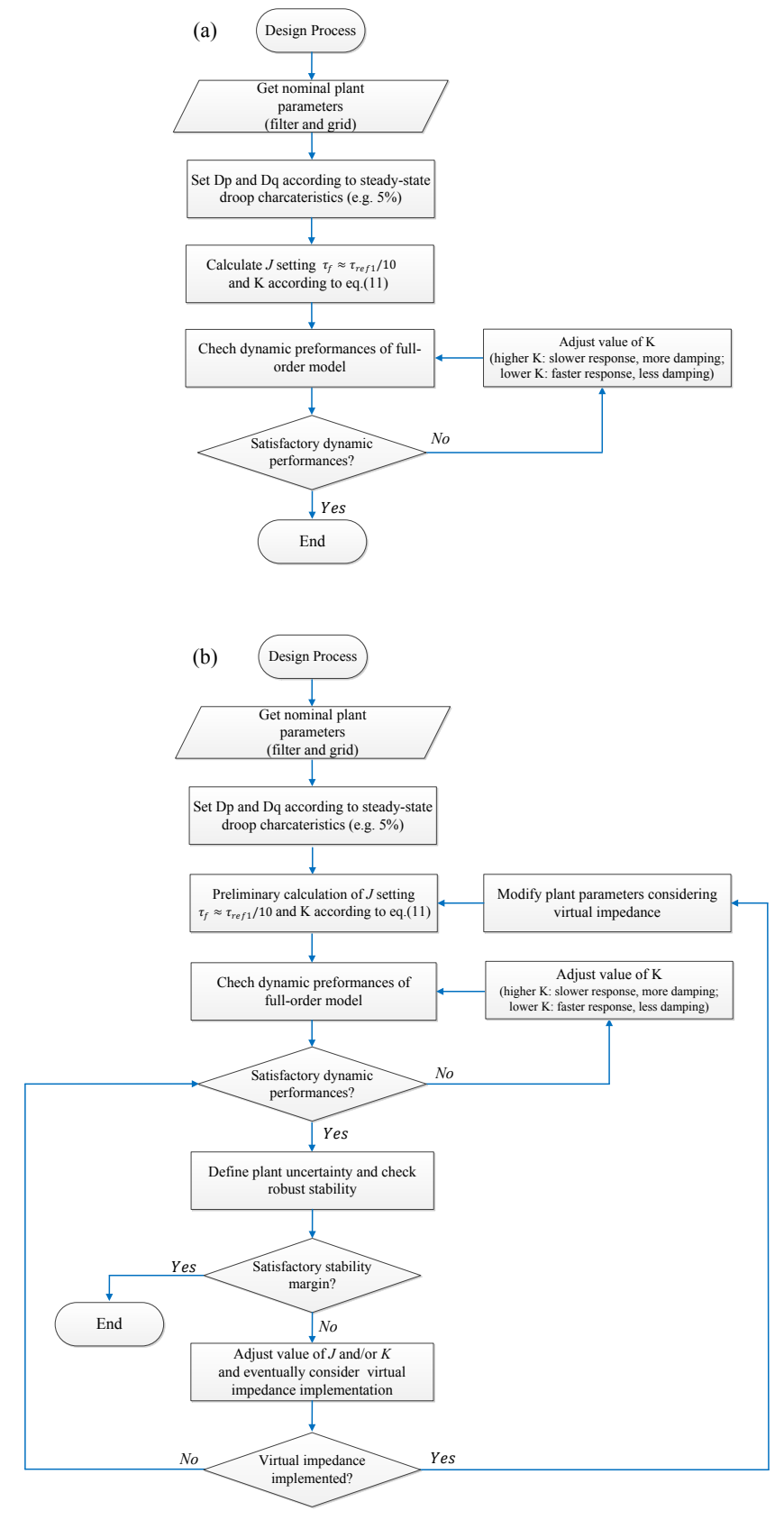

Fig. 15. (a) Steps of design procedure adopted in this paper and proposed in [10], (b) extended design procedure including robust stability considerations.

Danfoss of the Series FC-302 is connected to a 4-quadrant linear power amplifier PAS 15000 from Spitzenberger-Spies (single phase rated power $15 \mathrm{kVA}$, total three phase rated power $45 \mathrm{kVA}$ ). The converter is additionally equipped with a transformer to provide galvanic isolation.

The principle of PHIL simulations is briefly explained in the following. A virtual grid is simulated by means of a realtime simulator (RTDS in the specific case). The simulator measures the converter currents at the PCC, corresponding to the currents flowing into the power amplifier. In the RTDS, a grid model runs in real-time, simulating the effects of the currents injected by the converter on the virtual grid. The simulated voltages at the PCC are sent to the power amplifier as reference values $\widetilde{V}_{P C C}$. The linear power amplifier is able 
to reproduce at its terminal the reference voltages provided by the simulator almost instantaneously (slew rate $>52 \mathrm{~V} / \mu \mathrm{sec}$ ). This setup allows testing the behaviour of the converter under different grid conditions simply modifying the parameters of the grid model implemented in the simulator. The described setup has been used for validation of the model adopted for the analysis presented in this paper.

A resistive-inductive grid, as the one shown in Fig. 1, has been simulated in the RTDS and the parameters of the virtual grid have been modified so as to emulate a variation of the grid $S C R$ from 20 to 2 assuming a constant $X / R$ ratio of 10 . The control has been tuned following the procedure described in this paper. Setup parameters are shown in Table IV. In Fig. 17, measurements results are compared to timedomain simulations in MATLAB/Simulink/PLECS (where a voltage source has been used to model the converter) and to analytical results obtained from the small-signal model. Steps of active and reactive power of $0.25 \mathrm{pu}$ are shown for three different values of $S C R$, namely 20, 10 and 2, whereas the voltage control droop loop at the PCC has been either activated or deactivated. A good match between simulations and measurements can be appreciated. As already noticed in Fig. 5, the steady-state value of the output reactive power does not reach the given setpoint when the voltage droop is activated. This behaviour is much more accentuated for lower $S C R$. The $Q-V$ droop does not influence significantly the dynamic of the active power steps and therefore in Fig. 17 only $\frac{\Delta P}{\Delta P_{\text {set }}}$ steps for the case when the voltage droop is activated are shown.

Further experimental results are shown in Fig. 18, where
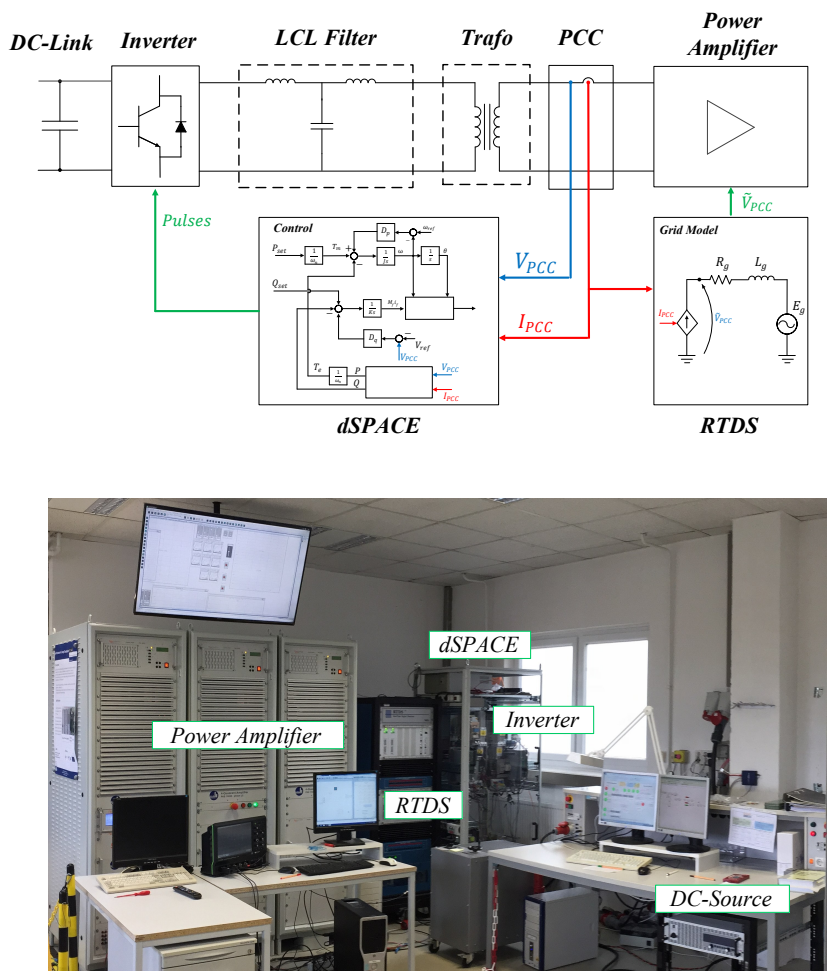

Fig. 16. (a) Scheme of the laboratory setup, (b) picture of the laboratory environment.
Table IV

PARAMETERS EXPERIMENTAL SETUP

\begin{tabular}{ccc}
\hline \hline Description & Symbol & Value \\
\hline Inverter rated power & $S_{n}$ & $4 \mathrm{kVA}$ \\
Line-to-line voltage & $V_{L L}$ & $400 \mathrm{~V}(\mathrm{rms})$ \\
Rated grid frequency & $f_{g}$ & $50 \mathrm{~Hz}$ \\
Inverter switching frequency & $f_{s w}$ & $5 \mathrm{kHz}$ \\
Inverter-side filter inductance & $L_{f}$ & $0.03 \mathrm{pu}$ \\
Grid-side filter inductance & $L_{f 2}$ & $0.003 \mathrm{pu}$ \\
Transformer inductance & $L_{T}$ & $0.003 \mathrm{pu}$ \\
Inverter-side filter resistance & $R_{f}$ & $0.0375 \mathrm{pu}$ \\
Grid-side filter resistance & $R_{f 2}$ & $0.018 \mathrm{pu}$ \\
Capacitor damping resistance & $R_{c}$ & $0.037 \mathrm{pu}$ \\
Filter capacitor & $C$ & $0.025 \mathrm{pu}$ \\
Virtual inertia & $J_{r e f}$ & $4 \mathrm{e}-4$ \\
Integrator gain & $K_{r e f}$ & 800 \\
P-Droop coefficient & $D_{p}$ & 0.679 \\
Q-Droop coefficient & $D_{q}$ & 183.71
\end{tabular}

\begin{tabular}{ccccc}
\hline Description & Symbol & SCR 20 & SCR 10 & SCR 2 \\
\hline Grid inductance & $L_{g}$ & $0.037 \mathrm{pu}$ & $0.075 \mathrm{pu}$ & $0.37 \mathrm{pu}$ \\
Grid resistance & $R_{g}$ & $0.0037 \mathrm{pu}$ & $0.0075 \mathrm{pu}$ & $0.037 \mathrm{pu}$ \\
\hline \hline
\end{tabular}

the parameters $J$ and $K$ have been varied from the reference values shown in Table IV. In Fig. 18 (b) and (e), the dynamic responses of the system to steps of $P_{\text {set }}$ and $Q_{\text {set }}$ of $0.25 \mathrm{pu}$ for $K=K_{r e f} / 8$ are respectively shown, whereas Fig. 18 (c) and (f) show the response of the system for $J=10 J_{\text {ref }}$.

Looking at the results shown in Fig. 17 and Fig. 18, it can be noticed how a decrease of the $K$ value causes a reduction of the high frequency damping of the system. An increase of the virtual inertia factor $J$ tends to slow down the system in terms of settling time and rise time. Additionally, an increase of the corresponding overshoot can be observed as well, indicating a reduction of low frequency damping. Operation of the converter has been tested even under extremely weak grid conditions $(S C R=2)$, which are typically critical for a standard grid connected converter. It can be noticed how in this case the response of the system is simply slowed down, but the light overshoot shown in the nominal case $(S C R=20)$ is eliminated instead. According to the presented results, one can conclude that the low frequency dynamic behaviour is not worsened by the lower $S C R$, which is instead the case for an increase of the virtual inertia $J$.

\section{CONCLUSiON}

In this paper, the robust stability analysis of a synchronverter connected to the grid through an output LCL filter is presented. A simple design procedure is introduced, which is used for tuning the control parameters according to nominal filter and grid characteristics. A robust stability analysis has been carried out based on the SSVs theory, which allows to assess the stability margin of the system according to a defined set of uncertainties. The effects of the variation of control parameters as well as grid characteristics on the stability of the system have been observed. Through the preformed $\mu$-analysis, some effects, which may not be clearly visible performing an eigenvalue analysis, have been highlighted. Among them, the fact that the synchronverter is more robust to uncertainties in the plant when connected to a grid with higher impedance is probably the most interesting. In fact, this behaviour goes 

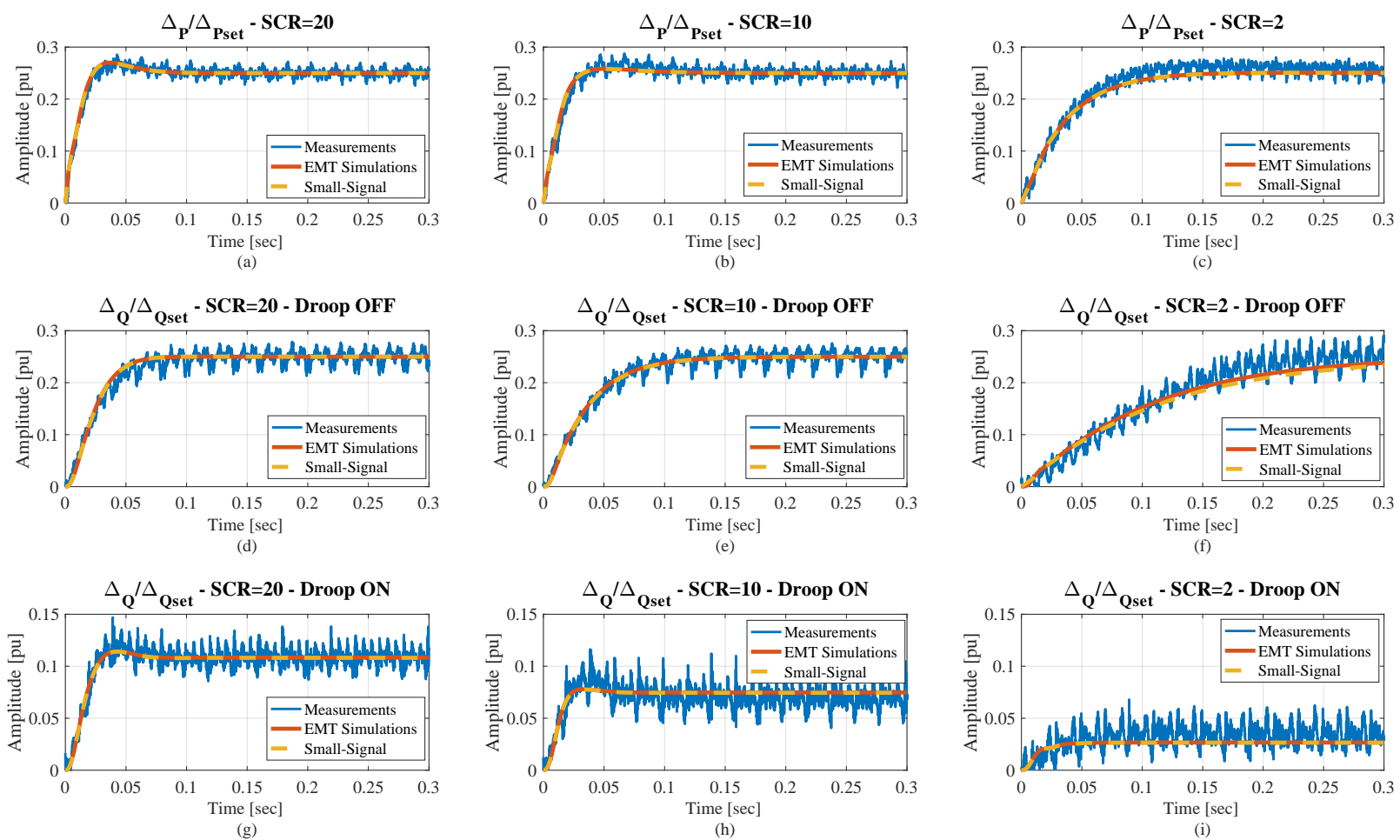

Fig. 17. Dynamic behaviour of $\frac{\Delta_{P}}{\Delta P_{s e t}}$, step of 0.25 pu: (a) $S C R=20$, (b) $S C R=10$, (c) $S C R=2$. Dynamic behaviour of $\frac{\Delta_{Q}}{\Delta Q_{s e t}}$, step of 0.25 pu, Q-V Droop off: (d) $S C R=20$, (e) $S C R=10$, (f) $S C R=2$. Dynamic behaviour of $\frac{\Delta_{Q}}{\Delta Q_{s e t}}$, step of 0.25 pu, Q-V Droop on: (g) $S C R=20$, (h) $S C R=10$, (i) $S C R=2$.

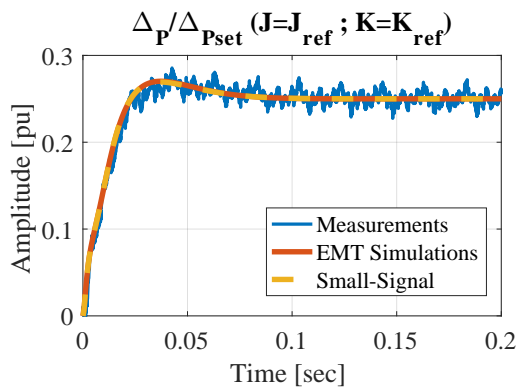

(a)

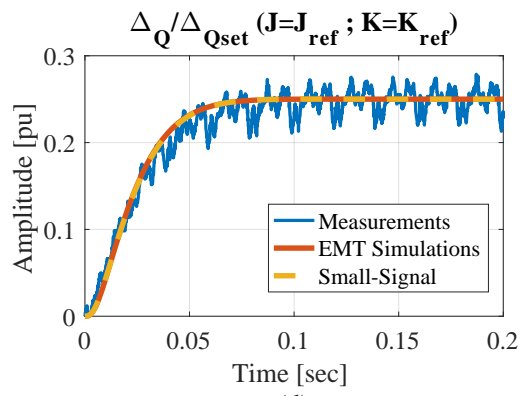

(d)

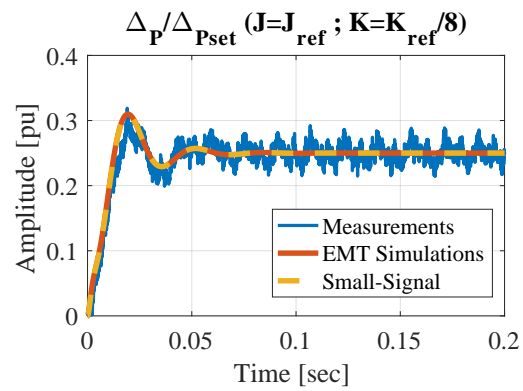

(b)

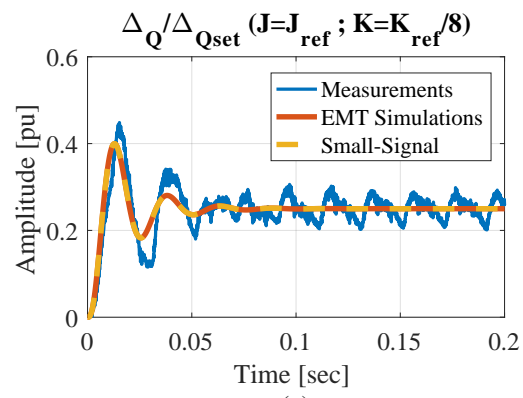

(e)

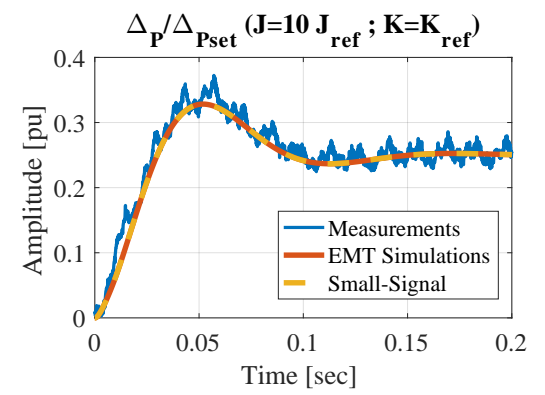

(c)

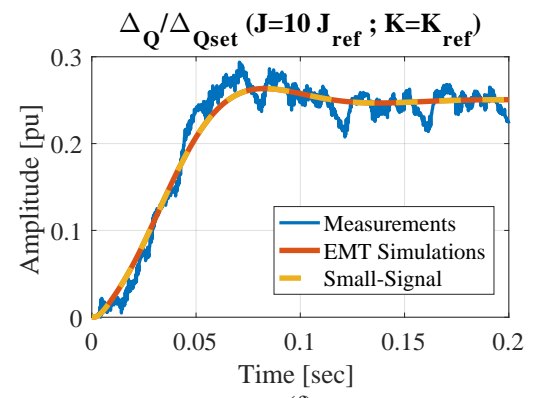

(f)

Fig. 18. Grid $S C R=20$, Q-V droop off, $\mathrm{X} / \mathrm{R}=10$. Dynamic behaviour of $\frac{\Delta_{P}}{\Delta P_{\text {set }}}$, step of $0.25 \mathrm{pu}$ : (a) $J=J_{\text {ref }}, K=K_{\text {ref }}$; (b) $J=J_{\text {ref }}, K=K_{\text {ref }} / 8$; (c) $J=10 J_{r e f}$, $K=K_{r e f}$. Dynamic behaviour of $\frac{\Delta_{Q}}{\Delta Q_{s e t}}$, step of 0.25 pu: (d) $J=J_{r e f}, K=K_{r e f}$; (e) $J=J_{r e f}, K=K_{r e f} / 8$; (f) $J=10 J_{r e f}, K=K_{r e f}$.

against the trend shown by grid connected converters using a dedicated synchronization unit. The model used for the analysis has been validated against simulations and laboratory experiments using a PHIL setup.

\section{REFERENCES}

[1] B. Wen, D. Boroyevich, R. Burgos, P. Mattavelli, and Z. Shen, "Analysis of D-Q small-signal impedance of grid-tied inverters," IEEE Trans. Power Electron., vol. 31, no. 1, pp. 675-687, Jan. 2016. 
[2] R. Rosso, G. Buticchi, M. Liserre, Z. Zou, and S. Engelken, ”Stability analysis of synchronization of parallel power converters," in Proc. 43rd Annual Conference of the IEEE Industrial Electronics Society (IECON), Beijing, China, 2017, pp. 440-445.

[3] H.-P. Beck and R. Hesse, "Virtual synchronous machine", in Proc. 9th Int. Conf. on Electrical Power Quality and Utilization (EQPU), Barcelona, Spain, Oct. 2007, pp. 1-6.

[4] S. D'Arco, J. A. Suul, and O. B. Fosso, "A virtual synchronous machine implementation for distributed control of power converters in smartgrids," Int. J. Electric Power System Research, pp. 180-197, 2015.

[5] Q.-C. Zhong and G. Weiss, "Synchronverters: inverters that mimic synchronous generators," IEEE Trans. Ind. Electron., vol. 58, no. 4, pp. 1259-1267, Apr. 2011.

[6] Q.-C. Zhong, P.-L. Nguyen, Z. Ma, and W. Sheng, "Self-synchronized synchronverters: inverters without a dedicated synchronization unit," IEEE Trans. Power Electron., vol. 29, no. 2, pp. 617-630, Feb. 2014.

[7] S. Dong and Y. C. Chen, "Adjusting synchronverter dynamic response speed via damping correction loop," IEEE Trans. Energy Convers., vol. 32, no. 2, pp. 608-619, Jul. 2017.

[8] H. Wu, X. Ruan, D. Yang, X. Chen, W. Zhao, Z. Lv, and Q.-C. Zhong, "Small-signal modeling and parameters design for virtual synchronous generators," IEEE Trans. Ind. Electr., vol. 63, no. 7, pp. 4292-4303, Jul. 2016.

[9] M. Amin, A. Rygg, and M. Molinas, "Self-synchronization of wind farms in an MMC-based HVDC system: a stability investigation", IEEE Trans. Energy Conv., vol. 32, no. 2, pp. 458-470, Jun. 2017.

[10] R. Rosso, J. Cassoli, S. Engelken, G. Buticchi, and M. Liserre, ”Analysis and design of LCL filter based synchronverter," in Proc. IEEE Energy Conv. Congr. and Exp. (ECCE), Cincinnati, OH, 2017, pp. 5587-5594.

[11] S. Dong and Y. C. Chen, "A method to directly compute synchronverter parameters for desired dynamic response," IEEE Trans. Energy Conv., vol. 33, no. 2, pp. 814-825, Jun. 2018 .

[12] R. Aouini, B. Marinescu, K. B. Kilani, and M. Elleuch, "Synchronverterbased emulation and control of HVDC transmission," IEEE Trans. Power Syst., vol. 31, no. 1, pp. 278-286, Jan. 2016.

[13] X. Wang, F. Blaabjerg, and W. Wu, "Modeling and analysis of harmonic stability in an AC power-electronics-based power system," IEEE Trans. Power Electron., vol. 29, no. 12, pp. 6421-6432, Dec. 2014.

[14] S. Skogestad and I. Postlethwaite, "Multivariable feedback control analysis and design", Wiley and Sons, 2001.

[15] K. Zhou and J. C. Doyle, "Essentials of robust control", Upper Saddle River: NJ. Prentice-Hall, 1998.

[16] L. Jessen, Z. Zou, B. Benkendorff, M. Liserre, and F. W. Fuchs, "Resonance identification and damping in AC-grids by means of multi MW grid converters," in Proc. 42nd Annual Conference of the IEEE Industrial Electronics Society (IECON), Florence, 2016, pp. 3762-3768.

[17] S. Sumsurooah, M. Odavic, and S. Bozhko, " $\mu$ approach to robust stability domains in the space of parametric uncertainties for a power system with ideal CPL ," IEEE Trans. Power Electron., vol. 33, no. 1, pp. 833-844, Jan. 2018.

[18] M. Liserre, F. Blaabjerg, and S. Hansen, "Design and control of an LCLfilter-based three phase active rectifier", IEEE Trans. Ind. Appl., vol. 41, no. 5, pp. 1281-1291, Sep. 2005.

[19] D. Schroeder, "Elektrische Antriebe 2, Regelung von Antriebssystemen", 2nd ed., Germany: Springer-Verlag, 2001.

[20] P. Kundur, "Power System stability and control", McGraw-Hill, Inc. 1994.

[21] G. J. Balas, J. C. Doyle, K. Glover, A. Packard, and R. Smith " $\mu$ Analysis and synthesis toolbox", Matlab user's guide.

[22] Z. Shuai, Y. Hu, Y. Peng, C. Tu, and Z. J. Shen, ’Dynamic-stability analysis of synchronverter-dominated microgrid based on bifurcation theory," IEEE Trans. Ind. Electr., vol. 64, no.9, pp. 7467-7477, Sep. 2017.

[23] V. Natarajan and G. Weiss, "Synchronverters with better stability due to virtual inductors, virtual capacitors and anti wind-up," IEEE Trans. Ind. Electron., vol. 64, no. 7, pp. 5994-6004, Jul. 2017.

[24] A. Terraso, J. I. Candela, J. Rocabert, and P. Rodriguez, "Grid voltage harmonic damping method for SPC based power converters with multiple virtual admittance control," in Proc. IEEE Energy Conv. Congr. and Exp. (ECCE), Cincinnati, OH, 2017, pp. 64-68.

[25] X. Lu, J. Wang, J.M. Guerrero, and D. Zhao, "Virtual impedance based fault current limiters for inverter dominated AC microgrids," IEEE Trans. Smart Grid, vol. 9, no. 3, pp. 1599-1612, May 2018.

[26] X. Wang, Y. W. Li, F. Blaabjerg, and P. C. Loh, "Virtual-impedancebased control for voltage-source and current-source converters," IEEE Trans. Power Electron., vol. 30, no. 12, pp. 7019-7037, Dec. 2015.

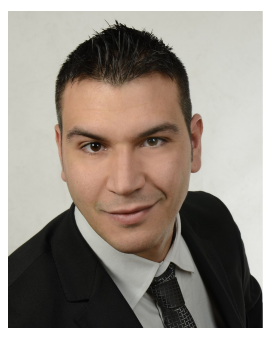

Roberto Rosso (S'17) received his B.Sc. in Electronic Engineering and his M.Sc. in Electrical Engineering in 2009 and 2012, respectively from the University of Catania, Italy. In 2013 he joined the R\&D division of the wind turbine manufacture ENERCON (Wobben Research and Development WRD), where he is currently working in the control engineering department. He has been involved in several research projects addressing analytical models of electrical machines and control of electric drive systems. Since 2017 he is pursuing the Ph.D. in electrical engineering at the Christian- Albrechts-University of Kiel, Germany. His research focuses on control strategies for grid integration of renewable energy systems.

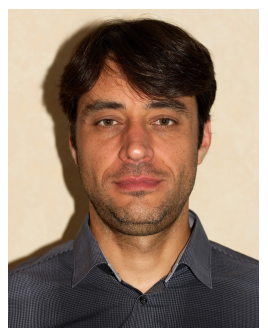

Jair Cassoli received in 2006 his M.Sc. in electrical engineering from the Technical University of Dresden, Saxony, Germany. He has been with ENERCON R\&D division in Aurich, Germany, a wind energy converter manufacturer, since 2006 as development engineer. $\mathrm{He}$ is responsible for model based design power control strategies for wind energy converters at the control system department. He has also been participating in collaboration with grid operators in several project specific studies concerning transient analysis of wind farm grid integration and protection based on EMTP modelling. His main research fields include control of electric machinery and drives for renewable energy systems and modelling and analysis of wind power plants connected to large power systems.

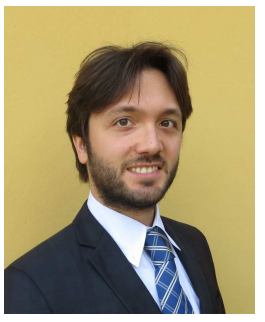

Giampaolo Buticchi (S'10-M'13-SM'17) received the Masters degree in Electronic Engineering in 2009 and the Ph.D degree in Information Technologies in 2013 from the University of Parma, Italy. In 2012 he was visiting researcher at The University of Nottingham, UK. Between 2014 and 2017 he was a post-doctoral researcher at the University of Kiel, Germany. He is now Associate Professor in Electrical Engineering at The University of Nottingham Ningbo China. His research area is focused on power electronics for renewable energy systems, smart transformer fed micro-grids and dc grids for the More Electric Aircraft. $\mathrm{He}$ is author/co-author of more than 150 scientific papers.

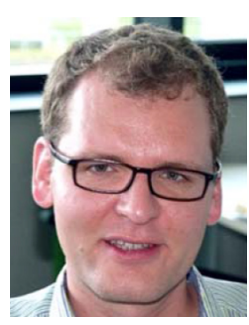

Soenke Engelken (S'08-M'12) is the Head of Control Engineering at WRD Wobben Research and Development. The Control Engineering department develops control solutions for wind energy converters, spanning wind turbine controls, electrical systems controls and grid-side converter controls. Prior to joining Wobben Research and Development, he received his Ph.D. and M.Sc. degrees in control engineering from the University of Manchester, UK, in 2012 and 2008, respectively, as well as his B.Sc. degree in electrical engineering and computer science from Jacobs University Bremen, Germany, in 2007. He is a member of the IEEE Power and Energy Society, the IEEE Control Systems Society, of CIGRÉ Joint Working Group A1/C4.52 Wind Generators and FrequencyActive Power Control and of the ENTSO-E Expert Group on High Penetration Issues.

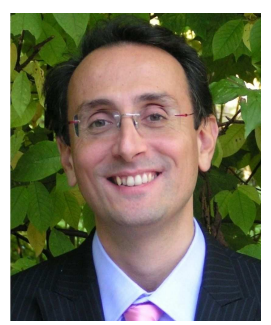

Marco Liserre (S'00-M'02-SM'07-F'13) received the $\mathrm{MSc}$ and $\mathrm{PhD}$ degree in Electrical Engineering from the Bari Polytechnic, respectively in 1998 and 2002. He has been Associate Professor at Bari Polytechnic and Professor at Aalborg University (Denmark). He is currently Full Professor and he holds the Chair of Power Electronics at ChristianAlbrechts-University of Kiel (Germany). He has published over 300 technical papers (more than 86 of them in international peer-reviewed journals) and a book. These works have received more than 20000 citations. Marco Liserre is listed in ISI Thomson report "The world's most influential scientific minds". 